\section{Atenção aos defeitos congênitos no Brasil: características do atendimento e propostas para formulação de políticas públicas em genética clínica}

\author{
Birth defects in Brazil and health care: \\ proposals for public policies in clinical genetics
}

\footnotetext{
${ }^{1}$ Instituto Fernandes Figueira, Fundação Oswaldo Cruz, Rio de Janeiro, Brasil. 2 Instituto de Medicina Social, Universidade do Estado do Rio de Janeiro, Rio de Janeiro, Brasil.

Correspondência D. D. G. Horovitz Centro de Genética Médica José Carlos Cabral de Almeida, Instituto Fernandes Figueira, Fundação Oswaldo Cruz.

Av. Ruy Barbosa 716, Rio de Janeiro, $R J$

22250-020, Brasil. dafne@ceresgenetica.com.br
} 
enfermidades crônicas degenerativas, fica ainda mais evidente o efeito dos condicionantes genéticos sobre a saúde ${ }^{1}$.

É fato documentado que as causas infecciosas como responsáveis pela taxa total de óbitos na infância vêm decaindo, resultando no aumento da proporção de mortes atribuíveis aos defeitos congênitos 2,3. Este dado também pode ser observado no Brasil, onde houve entre 1980 e 2000 grande redução proporcional dos óbitos por causas infecciosas e respiratórias, passando as malformações congênitas de quinta para segunda causa de mortalidade infantil 4,5,6.

Além da questão da mortalidade, há que se levar em conta a maior morbidade, definida como risco para o desenvolvimento de complicações clínicas, incluindo número de internações e gravidade das intercorrências. À medida que os problemas de origem infecto-contagiosa e carencial estão sendo resolvidos, aqueles de ordem congênita e hereditária se tornam pertinentes e de relevância na saúde pública, devendo ser alvo de ações oficiais específicas.

Dentro desse cenário, o objetivo deste trabalho foi mapear os serviços de genética em funcionamento no país para, a partir daí, avaliar formas de integrar e otimizar o atendimento aos defeitos congênitos na rede existente em genética clínica no Brasil.

\section{Material e métodos}

Foi elaborado questionário fechado, dentro de um modelo censitário, visando auferir quais os serviços públicos de genética clínica oferecidos à população brasileira, inclusive aqueles laboratoriais, integrados ou não a centros clínicos e instituições de ensino médico.

Aplicou-se o questionário a um subgrupo composto de chefes de serviços já mapeados no Estado do Rio de Janeiro, Brasil, para dimensionar a compreensão e ordem das perguntas formuladas. A realização desse pré-teste apontou para a necessidade de reformular-se oito questões no que tangia à ordenação e à terminologia empregada.

Em seguida empreendeu-se estudo piloto, tomando como população alvo chefes de serviços públicos de genética clínica sediados no Nordeste, no Sudeste e no Sul do país, a fim de corroborar a confiabilidade do instrumento.

Após essas etapas iniciou-se a coleta de dados, tendo como estratégia a utilização de formulário de autopreenchimento através de computador e/ou correio, a partir de listagem de serviços de genética no Brasil 7,8, cadastros de professores de genética de cursos de medicina 9 e relação de membros da Sociedade Brasileira de Genética Clínica (SBGC) e da Sociedade Brasileira de Genética (SBG). Cada questionário enviado era acompanhado de carta informando sobre a pesquisa, de acordo com o postulado pela Resolução n. 196/96 do Conselho Nacional de Saúde, e de pedido de divulgação entre os pares, requisitando a quem voluntariamente quisesse participar que entrasse em contato através de $e$-mail ou carta.

Os dados foram tabulados em planilha do programa Excel (Microsoft Corporation, Estados Unidos) e relacionavam-se a 60 serviços assim distribuídos: atendimento clínico + laboratório (33 instituições); atendimento clínico sem laboratório de genética (15 instituições); laboratório em genética humana não vinculado a atendimento clínico (8 instituições); ensino em genética, sem serviço clínico ou laboratorial (4 instituições).

A tabulação foi elaborada de modo a ter-se um mapa das principais características dos serviços, em especial quanto a: (a) oferta, (b) distribuição geográfica, (c) financiamento, (d) características do atendimento, (e) linhas de pesquisa e (f) projetos extramurais 5 . As informações obtidas foram comparadas às ações já existentes no país relacionadas aos defeitos congênitos 5,6, a dados demográficos 10 e a informações ambulatoriais e normas operacionais do Sistema Único de Saúde (SUS) 11,12 .

Para melhor expor e discutir os resultados obtidos subdividiu-se o presente artigo em dois itens principais. No primeiro descrevem-se as características dos serviços de genética no país, discutindo os principais problemas encontrados, ligados não apenas ao atendimento clínico, mas também ao fluxo de exames, referências e necessidades estimadas, e no segundo parte-se para a formulação de algumas propostas visando à integração da atenção aos defeitos congênitos no Brasil no âmbito do SUS. Reservou-se para as considerações finais a exposição de ações complementares recomendadas para efetivação e sedimentação dessa rede.

\section{Resultados e discussão}

\section{Características dos serviços de genética no Brasil}

\section{- Localização}

Responderam ao levantamento serviços de genética em 14 dos 25 Estados da União e no Distrito Federal. Na região Norte foi localizado apenas um serviço no Pará. No Nordeste responderam serviços no Ceará, Alagoas, Pernambuco, Bahia, Sergipe, Paraíba e Piauí. Os dados da região 
Centro-oeste vieram do Distrito Federal e Mato Grosso. Do Sudeste foram recebidas informações do Rio de Janeiro, São Paulo e Minas Gerais. Todos os Estados da região Sul tiveram representação nas respostas.

O único serviço na região Norte era exclusivamente laboratorial, embora dados mais recentes revelem atendimento clínico em genética já iniciado 13. No que tange à região Nordeste, os serviços são eminentemente clínicos, sem suporte laboratorial local em Pernambuco, Paraíba, Sergipe e Alagoas. O maior suporte laboratorial é na Bahia, havendo apenas um laboratório de citogenética no Ceará; por outro lado, o número de atendimentos anuais é significativo em alguns serviços da região. No Sudeste está concentrada a maioria dos serviços, tendo havido 34 respostas, sobretudo de São Paulo. Na região Sul observa-se cobertura clínico-laboratorial nos três estados. Tendo como principal exceção o Estado de São Paulo, a grande maioria dos serviços de genética clínica no Brasil está localizada nas capitais.

Através do levantamento, não obtivemos informações sobre serviços no Espírito Santo, Maranhão, Piauí e Rio Grande do Norte. Pelos dados disponíveis nos bancos de dados do Departamento de Informação e Informática do SUS (DATASUS) 11, no entanto, há indícios de serviços, tendo sido aprovados nesses estados números não desprezíveis de consultas em genética clínica provenientes das respectivas capitais. Nas cidades de Vitória (Espírito Santo), São Luis (Maranhão), Teresina (Piauí) e Natal (Rio Grande do Norte) há faculdades de medicina, além de hospitais universitários nas quatro localidades. Muito provavelmente os atendimentos em genética, nestes locais, estão vinculados aos serviços universitários. O fato de esses serviços não terem respondido ao levantamento reflete a desvinculação dos mesmos inclusive com a "rede informal” de serviços de genética existentes no país.

Na produção ambulatorial do SUS 11 é possível também localizar atendimentos em genética clínica em Campina Grande (Paraíba); Itajubá e Uberaba (Minas Gerais) e Joinville (Santa Catarina). Há faculdade de medicina em Campina Grande e Joinville, tendo sido localizados hospitais universitários em Campina Grande e Uberaba. Não se localizou nenhum dado relacionado a Itajubá. Além das consultas aprovadas, há cobranças de cariótipo (cariótipo em sangue periférico com técnica de bandeamento - código 1115101) em localidades ainda não mencionadas: Timóteo (Minas Gerais); Caraguatatuba, Presidente Prudente, Santos e São Vicente (São Paulo); Cascavel, Foz do Iguaçu e Umuarama (Paraná); Blumenau (Santa Catarina); Rio do Sul, São Bento do Sul e São José (Rio Grande do Sul);
Campo Grande e Dourados (Mato Grosso do Sul). Tais cariótipos podem estar sendo realizados sem vinculação com serviços de genética nestas localidades.

\section{- Atendimentos}

Segundo dados fornecidos pelos serviços, são estimadas anualmente cerca de 56 mil consultas ambulatoriais em genética clínica no país (incluindo atendimentos a casais - aconselhamento genético - e pré-natais). Desse montante, pouco menos de 16 mil representam casos de primeira vez. Vale ressaltar que do total de atendimentos, mais da metade são realizados em nove instituições apenas.

\section{a) Consultas oferecidas $x$ estimativa de necessidade}

Caso seja considerado que todo recém-nascido com malformação detectada ao nascimento tenha indicação de avaliação por geneticista, com uma incidência de malformações minimamente estimada ( $2 \%$ do total de nascidos vivos), seriam necessárias 64.102 consultas/ano no país para casos de primeira vez. A estimativa é mais deficitária se considerada a definição mais ampla de defeitos congênitos, incluindo problemas não necessariamente detectados ao nascimento e alterações do desenvolvimento (nesse caso, 5\% do total de nascimentos representariam 160.255 novos casos/ano). É também válido lembrar que muitos atendimentos relacionados a defeitos congênitos são conseqüência de insucessos gestacionais, sendo indicado o aconselhamento genético a pais de natimortos com malformação, não incluídos nestas estimativas. Analisando esses dados, o número de consultas ofertadas está muito abaixo da real necessidade do país.

Visando ilustrar a relação entre a oferta de atendimentos em genética clínica e a demanda estimada, foi construída a Tabela 1, com o número de nascimentos por região, estimativa de bebês com defeitos congênitos, oferta de consultas de primeira vez nos serviços pesquisados e número de consultas aprovadas em genética clínica (código 0701216) da produção ambulatorial do SUS. Em todas as regiões, sem exceção, há um déficit óbvio de consultas, em especial para atendimentos de primeira vez, mesmo se considerados como clientes em potencial para os serviços de genética apenas $2 \%$ dos recém-nascidos. As regiões mais críticas são Norte e Nordeste. No Centro-oeste a totalidade dos atendimentos é realizada em Brasília. É importante lembrar que as consultas não foram apenas de casos novos, tendo os serviços estimado os casos de primeira 
Número de nascimentos, estimativa de defeitos congênitos (considerando $2 \%$ e $5 \%$ do total de nascidos vivos), estimativa de consultas em genética clínica - número total e pacientes de primeira vez ${ }^{*}$, consultas em genética clínica pagas. Dados estratificados por regiões.

\begin{tabular}{|c|c|c|c|c|c|c|}
\hline Região & $\begin{array}{l}\text { Nascimentos } \\
(2000)^{* *}\end{array}$ & $\begin{array}{c}2 \% \text { dos } \\
\text { nascimentos }\end{array}$ & $\begin{array}{c}5 \% \text { dos } \\
\text { nascimentos }\end{array}$ & $\begin{array}{l}\text { Consultas na } \\
\text { região } \\
\text { (estimativa dos } \\
\text { serviços) }\end{array}$ & $\begin{array}{l}\text { Consultas de } \\
\text { primeira vez } \\
\text { (estimativa dos } \\
\text { serviços) }\end{array}$ & $\begin{array}{c}\text { Consultas em } \\
\text { genética clínica - } \\
\text { quantidade } \\
\text { paga }\end{array}$ \\
\hline Norte & 290.546 & 5.811 & 14.527 & 0 & 0 & 0 \\
\hline Nordeste & 925.681 & 18.513 & 42.284 & 8.065 & 1.952 & 11.569 \\
\hline Centro-oeste & 232.587 & 4.651 & 11.629 & 4.420 & 1.000 & 5.528 \\
\hline Sudeste & 1.306 .113 & 26.122 & 65.305 & 35.363 & 10.677 & 30.064 \\
\hline Sul & 450.181 & 9.003 & 22.509 & 8.267 & 1.816 & 6.961 \\
\hline Brasil & 3.205 .108 & 64.102 & 160.255 & 56.604 & 15.684 & 54.122 \\
\hline
\end{tabular}

* Horovitz 5 ;

** Sistema de Informações de Saúde. Sistema de Informações Sobre Nascimentos (SINASC) 10;

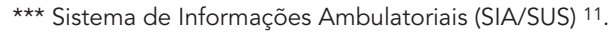

vez como $28 \%$ do total das consultas realizadas 5 . Na hipótese de considerar como potenciais candidatos à avaliação por geneticista clínico $5 \%$ do total de nascidos vivos (cerca de $160 \mathrm{mil} / \mathrm{ano}$, na definição mais ampla de defeitos congênitos), menos de $10 \%$ desses pacientes estão conseguindo acesso aos serviços (Tabela 1).

\section{b) Problemas encontrados nos serviços de genética}

Algumas questões relacionadas ao funcionamento dos serviços, principalmente no que diz respeito ao acesso, fluxo e integração com a rede básica devem sem discutidas.

\section{(i) Acesso}

Em alguns dos serviços, sobretudo nos maiores, o agendamento da consulta inicial pode demorar alguns meses. No levantamento realizado, apesar de vinte serviços referirem intervalo entre marcação e primeira consulta de até um mês, apenas dois destes têm volume superior a 2 mil atendimentos/ano. Nos três serviços com mais mil casos novos/ano (Instituto Fernandes Figueira, Fundação Oswaldo Cruz - Rio de Janeiro; Escola Paulista de Medicina, Universidade Federal de São Paulo - São Paulo; Universidade Estadual de Campinas - São Paulo), o intervalo entre o agendamento e a primeira consulta pode chegar a um ano, sendo raramente inferior a dois meses.

Devido à espera muitas vezes longa para atendimento na especialidade, algumas famílias buscam múltiplos serviços, principalmente quando há maior oferta na região de residência. É feito agendamento de consulta em mais de uma instituição, com comparecimento àquela que ofereceu o atendimento com menor prazo de espera. Apesar de já obtida inserção na rotina de serviço especializado, as marcações nos demais serviços não são canceladas. Por este motivo, é muito freqüente observar faltas em consultas de primeira vez agendadas com antecedência, o que dificulta ainda mais o acesso aos serviços de genética.

Além das múltiplas tentativas de atendimento dentro da região de residência, algumas famílias o buscam fora de domicílio, eventualmente por conta própria, tornando ainda mais confuso o acesso à especialidade e dificultando a remuneração junto ao SUS. Estes atendimentos fora de domicílio ocorrem principalmente em serviços muito especializados, como o Hospital de Reabilitação de Anomalias Craniofaciais/Bauru, centro de referência em lesões lábio-palatais, que tem mais de $50 \%$ de seus pacientes originários de outros Estados 5

\section{(ii) Dificuldades de referência/ contra-referência}

Além das dificuldades inerentes ao acesso aos serviços, há alguns obstáculos relacionados à contra-referência daqueles pacientes já atendidos. De um modo geral, observa-se que o acesso aos serviços de genética é difícil, porém, quando obtido, pais e outros profissionais acabam criando um "vínculo psicológico" com a instituição, dificultando a contra-referência para o local de origem.

Não há como viabilizar, nos serviços de genética clínica, o acompanhamento periódico de 
todos os pacientes com defeitos congênitos. Muitas vezes há recusa dos serviços básicos de saúde em prestar atendimento aos mesmos, alegando "desconhecimento" sobre a condição. O diferencial do geneticista em relação a outros profissionais médicos é o estabelecimento do diagnóstico, fornecimento de orientações prognósticas e recomendações para acompanhamento, além do aconselhamento genético da família. Após a investigação, com ou sem diagnóstico conclusivo, uma vez traçadas as estratégias de acompanhamento, deverá ser fornecido relatório completo, para que o paciente possa ser seguido próximo ao domicílio. Importante ressaltar que os serviços de referência podem ser consultados sempre que considerado necessário pelo paciente, sua família ou equipe de saúde responsável pelo acompanhamento.

\section{(iii) Concentração dos serviços nas capitais grandes áreas urbanas}

Tendo como principal exceção o Estado de São Paulo, com serviços em diversas cidades fora da capital, a maioria dos serviços de genética clínica no Brasil está localizada nas capitais estaduais. Tal característica não é restrita aos serviços de genética, aplicando-se a serviços de maior complexidade em geral. Por este motivo, o acesso ao atendimento é sempre mais difícil, principalmente para a população de áreas mais remotas, envolvendo grandes deslocamentos.

\section{(iv) Pacientes com defeitos congênitos inter- nados em unidades sem geneticista}

São estimadas anualmente pelos serviços 3.400 consultorias hospitalares (avaliação de pacientes durante internação hospitalar por especialista em genética) na especialidade 5. Quando comparado este dado com a proporção anual de nascimentos com malformação congênita (Figura 1), é possível inferir que a maioria dos pacientes nascidos em hospital sem serviço de genética não é avaliada antes da alta hospitalar. Não há previsão de visitas hospitalares em hospitais da rede por geneticistas vinculados a outras instituições. A falta de avaliação especializada freqüentemente acarreta condutas inadequadas de tratamento, podendo inclusive resultar em maiores custos hospitalares. Muitos hospitais da rede pública, das capitais ou grandes regiões metropolitanas, optam por levar pacientes internados para avaliação ambulatorial em instituição com geneticista, sendo necessária disponibilidade de pessoal e transporte.

Ressalta-se que muitos dos bebês nascidos com defeitos congênitos evoluem para o óbito rapidamente, sequer chegando a ser avaliados em vida por especialista. Além disso, há poucos serviços capacitados em patologia fetal e neonatal, sendo escassas as necrópsias realizadas, dificultando sobremaneira a orientação das famílias e prevenção de futuros casos.

\section{(v) Vinculação dos serviços de genética clínica}

Talvez uma das grandes dificuldades para a cobertura do atendimento aos defeitos congênitos no Brasil e do estabelecimento de uma rede de genética clínica seja a não oficialização do cargo de "médico geneticista" no sistema público de saúde 14. Apesar de ser possível, dentro da política do SUS, a criação de tal cargo, não tem havido contratação de profissionais desta área. Algumas justificativas seriam as próprias características da especialidade (relativamente nova, multidisciplinar em essência, origem heterogênea dos serviços), além da errada impressão que a especialidade não pode atuar na atenção básica ou desprovida de alta (e cara) tecnologia.

Quando utilizados os dados obtidos no levantamento 5 , é importante apontar que, dentre os serviços de genética que oferecem atendimento clínico (48 no total), apenas quatro não possuem vínculo com instituição de ensino médico (graduação, residência médica, especialização ou pós-graduação). Também é possível observar a dificuldade do estabelecimento de serviços de genética como genética propriamente, mesmo em ambientes de ensino, onde quase metade tem seu atendimento clínico em genética vinculado a outras áreas (pediatria, clínica médica ou endocrinologia). Nessas instituições, muitos dos que atuam na especialidade acabam fazendo-o como "desvio de função", freqüentemente acumulando outras atribuições, desperdiçando parte da capacidade que os diferencia e que certamente poderia ser mais bem aproveitada.

\section{- Laboratórios de genética médica e rede laboratorial}

A Genética Médica é multidisciplinar em essência, assim como colaborativa. Lidar com condições raras é característica da especialidade, sendo impossível manter suporte laboratorial para diagnóstico de todas as condições. No mundo inteiro, laboratórios vêm especializando-se em diagnósticos específicos, não sendo tal fato diferente no Brasil.

No levantamento sobre os serviços, alguns referem possuir apenas laboratório de citogenética, enquanto outros oferecem diagnósticos mais específicos de genética molecular, citogenética 
molecular e erros inatos do metabolismo. No entanto, pelas próprias características da genética clínica, muitas vezes é necessário encaminhar amostras para diagnóstico a laboratórios em outros estados ou até mesmo em outros países.

Exames enviados para fora das respectivas unidades, mesmo que dentro do próprio Estado, ou para outros Estados da União, na maioria das vezes não são pagos ao laboratório que presta o serviço. Segundo informação das instituições que oferecem esses exames, não há mecanismos previstos para cobrança deste tipo de serviço (exames para outras instituições/município/Estado). Como formas de pagamento, foram citadas no levantamento: cobrança direta aos usuários (pacientes), pagamento pelo próprio departamento/serviço que envia o exame, utilização de verbas de pesquisa, uso de reserva técnica de projetos, estudos colaborativos e até "vínculo de amizade/boa vontade do dirigente do laboratório" 5 . Apesar de inúmeros casos serem de interesse científico, há custos a cobrir. Pela ausência de mecanismos para tais cobranças, grande parte do intercâmbio entre serviços e laboratórios é baseada apenas no conhecimento pessoal e boa vontade.

Há no Brasil uma rede informal de referências laboratoriais em genética, e quase uma dezena de laboratórios recebem amostras para análises especiais. Dentre estes, foi citado por 25 serviços de todas as regiões brasileiras o Serviço de Genética Médica, Hospital de Clínicas de Porto Alegre/Universidade Federal do Rio Grande do Sul, referência nacional reconhecida para erros inatos do metabolismo. Serviços do Rio de Janeiro e São Paulo também foram mencionados como referência para diagnósticos moleculares e citogenética especializada 5 .

\section{Propostas para a organização da genética} clínica no Brasil como uma rede funcional

Considerando a crescente magnitude dos defeitos congênitos nas questões da saúde da população, é recomendável que em futuro não muito distante sejam consideradas estratégias para a sua abordagem. Para a estruturação de um programa ou estratégia específica, é prioritária a organização dos serviços de genética clínica do país de forma racional, visando à ampliação das possibilidades de atendimento, integração de todas as etapas do diagnóstico e resolubilidade dos casos. É premente ressaltar que a elaboração de uma estratégia não necessariamente envolve instalação de muitos serviços ou aumento de custos, mas sobretudo o aproveitamento da rede já existente, fundamentada em organização planejada.
A discussão de regionalização é a mais importante quando objetivada uma assistência integral às enfermidades congênitas. Dentro da lógica do SUS, a fragmentação da gestão para os municípios pode ser excelente na atenção à saúde em inúmeros aspectos; no âmbito dos defeitos congênitos, contudo, lida-se com doenças raras e cuidados heterogêneos. A organização da genética clínica no sistema de saúde brasileiro possivelmente transcende às gestões municipais, tendo, no entanto, boas possibilidades de sucesso, caso coordenada por instância superior, como o Ministério da Saúde. Uma rede de genética clínica poderia funcionar com duas vertentes de organização: a primeira uma rede integrada de atendimento clínico, e a segunda, uma rede de suporte laboratorial. Propostas para a estruturação de rede nacional em genética clínica, juntamente com sugestões visando à resolução de questões levantadas no início do texto serão discutidas a seguir.

\section{- Rede para atendimento clínico em genética}

Esta poderia ser estruturada de acordo com cada Estado, município ou região, critério a ser estabelecido pela população a ser atendida, necessidade e disponibilidade de serviços na área. O objetivo de uma rede clínica seria a integração de todos os serviços existentes, complementando-se, além do reconhecimento da importância da especialidade pelas autoridades de saúde, disponibilizando atendimento na área, tanto hospitalar quanto ambulatorial. Para tal, seriam necessários geneticistas com vínculo não exclusivamente institucional, e sim com o sistema.

\section{a) Sistema de agendamento}

Caso possível, a integração de serviços de genética de uma mesma região em uma rede única, poderá ser considerada um sistema único de agendamento para consultas de la vez. Com tal medida, seria combatida a duplicidade de marcações, melhorando assim o acesso aos serviços e diminuindo o tempo de espera para a consulta inicial.

\section{b) Centros de referência}

Há duas maneiras de interpretar o termo "centro de referência”. A primeira e mais óbvia seria considerar tratar-se de local para atendimento a questões de maior complexidade, dispondo de todos os recursos para resolvê-las. Dentro desta definição, se considerarmos como essencial para centro de referência em genética clínica o 
atendimento nas áreas de dismorfologia, retardo mental, erros inatos do metabolismo e aconselhamento genético, além de exames de citogenética, erros inatos do metabolismo e biologia molecular, menos de dez serviços preencheriam tais critérios 5 . Caso o diagnóstico pré-natal seja também considerado como pré-requisito, seriam apenas cinco os serviços "completos" no país.

A segunda interpretação seria um pouco mais flexível quanto aos pré-requisitos para centro de referência: poderia ser considerada a especialização clínica de cada centro, e não necessariamente os exames oferecidos. Considerando no Brasil uma estruturação dos serviços de genética em rede, na impossibilidade de aparelhar os serviços visando ampliar o diagnóstico laboratorial, há opções para arranjo de referências. Pólos de referência por área geográfica podem ser criados, de acordo com as particularidades de cada região, muito provavelmente transcendendo regiões administrativas municipais e/ou estaduais. A referência seria pelo perfil do paciente a ser atendido: centros especializados para lidar melhor com diagnósticos específicos (fendas lábio-palatinas, erros metabólicos, distrofias musculares, por exemplo); ou mesmo centros para suporte diagnóstico, com disponibilidade de métodos diagnósticos gerais (radiologia, ecocardiograma, por exemplo) e especialistas em diversas áreas. De acordo com esta visão, caso considerada ampliação da rede em genética além dos serviços computados no levantamento 5 , os hospitais universitários seriam locais ideais para o estabelecimento de tais centros. Segundo Brunoni 15, o panorama da assistência em genética médica no Brasil começará a mudar caso seja criada pelo menos uma unidade de genética em cada um dos 150 hospitais universitários do país.

A possibilidade de internação nos centros de referência é altamente recomendada mesmo que apenas para investigação diagnóstica em casos mais complexos. Assim, exames e avaliações especializadas podem ser concentrados em menos tempo, com menor sacrifício das famílias. Todas as ações precisariam ser integradas pela equipe médica e de serviço social. Ações competentes de especialistas acabam se perdendo em sistemas deficientes, com grande prejuízo e sofrimento do cidadão.

Outra atribuição dos centros de referência seria o tratamento e a monitorização de condições específicas. Um exemplo de tratamento que o geneticista pode oferecer é para o grupo dos erros inatos do metabolismo. A raridade dessas condições e a complexidade das terapias dietéticas justificam um acompanhamento por centros de referência em genética 16 . Além das questões já levantadas, devemos ressaltar a disponibilidade de tratamento para algumas doenças geneticamente determinadas, com estratégias terapêuticas recentes e estranhas à maioria dos profissionais da área médica. A centralização do tratamento dessas doenças raras permitiria a formação de pessoal capacitado, criação de leitos especialmente voltados para este fim, estabelecimento e sedimentação de protocolos. Uma vez bem estabelecidos, os protocolos poderão ser adotados por outras unidades. Tratamentos para doença de Gaucher e osteogênese imperfeita são bons exemplos de otimização pela centralização 5 .

\section{c) Geneticista itinerante}

O reconhecimento de indivíduos ou famílias com malformação congênita ou patologia hereditária, muitas vezes apenas complementado com histórico familiar detalhado e avaliação clínica básica por especialista capacitado podem freqüentemente ser suficientes para estabelecimento de diagnóstico e conduta. Uma abordagem clínica inicial próxima ao domicílio do paciente pode, além de evitar grandes deslocamentos, ser totalmente resolutiva. É possível criar estratégias para avaliação de grupos de pacientes em locais próximos aos domicílios através da figura do "geneticista itinerante”. Este profissional, vinculado ao sistema de saúde (e não a uma unidade específica), poderia atender com periodicidade pré-estabelecida, de acordo com a necessidade do local (semanal, mensal ou bimensal, por exemplo, cobrindo várias localidades), em pólos de atendimento clínico, como cidades com hospitais universitários ou faculdades de medicina.

Ainda na filosofia do profissional em genética clínica "itinerante", geneticistas consultores poderiam atuar auxiliando equipes no delineamento de condutas e tomada de decisões. Esses especialistas poderiam ser responsáveis por múltiplas unidades de internação pediátricas e neonatais, prestando consultoria em genética e levando a uma maior resolubilidade dos casos. Um pré-requisito para este tipo de ação seria a vinculação, no SUS, de médicos geneticistas à rede de atendimento, com a criação de cargo que possibilite o perfil sugerido de consultor para múltiplas instituições. Para funcionamento ideal das consultorias hospitalares por geneticista, deverá também ser estabelecida rede clínica e laboratorial de suporte.

\section{d) Introdução da genética na atenção primária}

A inserção da "mentalidade genética" na atenção primária seria a ação mais eficaz para a implantação de uma rede efetiva. O papel dos profissio- 
nais, no nível primário, incluiria a identificação, referência e facilitação da utilização dos serviços para indivíduos que possam ser beneficiados pelo atendimento em genética, além do monitoramento da saúde de pessoas com problemas genéticos 17 .

O paciente com defeito congênito pode e deve ser acompanhado próximo ao seu domicílio, não havendo assim prejuízo ainda maior na qualidade de vida. Para um encaminhamento correto, o profissional da atenção básica deverá estar capacitado para reconhecer a pessoa com defeito congênito e famílias em risco. A partir desse reconhecimento, estratégias de atendimento, próximas ao domicílio ou em centro de referência, poderão ser programadas.

Apesar do objetivo principal deste artigo não ser a introdução da genética na atenção primária e sim a estruturação da rede de atenção aos defeitos congênitos como um todo, deve-se ressaltar a importância da atenção básica no processo. Estas unidades, por serem porta de entrada no sistema, podem incorporar estratégias cruciais para a prevenção dos defeitos congênitos, conforme preconizado por Boulyjenkov 18 . O suporte à triagem neonatal pela atenção básica, incluindo informação genética, vem sendo inserido de forma eficaz no país 5,6. A incorporação de outras ações ao nível primário, como programas educativos, rastreamento de gestantes/casais com alto risco reprodutivo e busca ativa de pessoas em risco para defeitos congênitos pode ser considerada 19 . É sempre válido ressaltar a importância da integração dessas estratégias ao plano de ação para a abordagem dos defeitos congênitos no país.

\section{- Rede laboratorial de suporte para genética clínica}

Para que a genética clínica seja formalizada como uma rede funcional no âmbito do SUS, deverão ser avaliadas as particularidades da especialidade, visando estruturar coberturas clínica e laboratorial, possivelmente independentes, embora complementares. A rede poderá ser regionalizada e hierarquizada no âmbito do atendimento clínico, embora muito provavelmente não municipalizada, nem necessariamente restrita ao Estado em certas regiões. Há três tipos freqüentes de testes genéticos: citogenético (análise cromossômica), testes moleculares e análises bioquímicas. Quanto à cobertura laboratorial, no entanto, para uma rede funcional e economicamente viável, não seria justificada a implantação de laboratórios especializados em todos os serviços ou mesmo em todos os Estados da União. Laboratórios básicos de citogenética e triagem de erros metabólicos poderiam ser implemen- tados em nível estadual ou regional, visando dar cobertura aos serviços de cada região. Por outro lado, análises mais especializadas (bioquímicas/ moleculares) poderiam ser oferecidas por poucos centros de excelência. $\mathrm{O}$ financiamento de tais exames precisaria ser revisto, uma vez que referências laboratoriais inter-Estado não estão previstas na organização do SUS 12. Tal organização também não contempla o fluxo de exames, apesar da possibilidade do fluxo de pacientes em situações consideradas de altíssima complexidade. Ressalta-se que na genética, alta complexidade não é sinônimo de alto custo, embora esses dados já indiquem claramente que a organização de rede na especialidade deva ser nacional.

Organizar a rede laboratorial básica e de suporte e formalização da referência é um dos passos mais importantes para o bom funcionamento da rede de genética no país. Deve-se lembrar que é necessário prever não apenas o financiamento de exames, mas também o transporte de amostras, geralmente possível via correio, porém não incluído em previsões de custos laboratoriais. Este fluxo é muito mais simples, barato e menos traumático para as famílias do que a peregrinação de pacientes em busca de mais recursos diagnósticos.

\section{- Complementação adicional e suporte à rede de genética clínica}

Mais uma vertente sugerida para a organização da genética clínica em rede no Brasil funcionaria como complementação das redes clínica e laboratorial, fornecendo suporte técnico relacionado a auxílio diagnóstico e orientações quanto a questões mais específicas. Como exemplos de serviços especiais de suporte podem ser citados os de informação relacionados a agentes teratogênicos (SIATs) e serviços de suporte para condições específicas, como por exemplo o SIEM (que fornece orientação de condutas quanto aos erros inatos do metabolismo) 5,6. Uma rede de apoio, de especial relevância para suporte clínico a regiões mais remotas, também poderia incluir grupos de geneticistas bastante experientes para consultoria, utilizando a internet para contatos e orientações.

\section{Considerações finais}

Além de formalizar e efetivar a organização de uma rede de genética clínica no Brasil, outras ações precisarão ser implementadas para que o sistema não apenas funcione adequadamente, mas que também seja progressivamente expandido e adaptado de acordo com as necessidades 
crescentes do país. Iniciativas governamentais já existentes deverão ser sedimentadas, e programas não-governamentais poderão eventualmente ser agregados ao sistema, visando seu enriquecimento e otimização. Tópicos de relevância para a efetivação do atendimento aos defeitos congênitos são apresentados abaixo.

\section{Registro de malformações congênitas}

Deve ser estimulada a implementação efetiva do "campo 34" nas declarações de nascimento (que registra a presença de malformação congênita no recém-nascido) 20 , através de um trabalho de base com as prefeituras, visando seu preenchimento correto. Tais dados serão de grande relevância para o acompanhamento de incidências dos defeitos congênitos, programação de ações de saúde específicas e avaliação da eficácia de ações já introduzidas.

\section{Estímulo a organizações de pais e pacientes}

Esses grupos, além de possibilitar um maior suporte e mesmo conforto aos seus membros, têm como meta a divulgação de informação, inclusive à classe médica, além de poderem vir a exercer papel fundamental para a introdução de novos temas na agenda política 21 .

\section{Educação}

Apesar de cada vez mais presente no dia-a-dia da prática médica, a genética médica (disciplina diferenciada da genética celular e molecular básica) não figura como matéria no currículo mínimo dos cursos de graduação em Medicina 7. Por este motivo, muitos médicos desconhecem completamente a especialidade, ignorando as possibilidades de atendimento clínico em genética.

\section{Pesquisa}

A pesquisa em genética nos países em desenvolvimento deve concentrar-se, inicialmente, em proporcionar melhor conhecimento epidemiológico sobre as doenças genéticas e os defeitos congênitos 1,21. Estudos que busquem o diagnóstico da situação dos serviços e que levantem as necessidades da clientela seriam itens essenciais a serem pesquisados 22 . Resultados de levantamento com este teor já estão disponíveis ${ }^{5}$. A partir da caracterização dos serviços, complementada por dados epidemiológicos, torna-se viável o planejamento de uma estratégia para a assistência integral em genética. Devem também merecer especial atenção os métodos de educação pública em genética e a avaliação das estratégias de prevenção dos defeitos congênitos a serem lançadas 22 .

\section{Prevenção}

Poderíamos ter páginas de discussão sobre o que seria aceitável e o que não seria considerado ético na área de prevenção. Obviamente, para várias situações em genética, a detecção precoce (em alguns casos, na gravidez) é a única forma de prevenir o nascimento de uma criança afetada. É fundamental ressaltar que não raro os profissionais, geneticistas ou não, deparam-se com questões de grande complexidade ética e legal. Nesse momento, é fundamental que se retorne ao tópico da educação. Decisões complexas só deveriam partir de uma real compreensão dos fatos, assim como dos direitos de cada um. A genética clínica é defensora da autonomia das famílias em suas decisões.

\section{Formalização da genética clinica no SUS}

A figura do geneticista clínico não vinculado a centro de referência ou universitário praticamente inexiste no SUS. São raros os concursos públicos para médico geneticista, e profissionais capacitados acabam trabalhando em outras especialidades por falta de oportunidade. O reconhecimento e formalização da genética clínica como especialidade médica na rede pública de saúde, conforme preconizado por Faria et al. 14, permitirá a criação de novos pólos de atendimento clínico e difusão do conhecimento entre profissionais de saúde, aumentando a captação de novos casos e agindo de forma positiva no diagnóstico, tratamento e prevenção dos defeitos congênitos.

Dados já levantados 5 poderão ser utilizados como base para estruturar uma ação relacionada aos defeitos congênitos, desencadeando um efeito transformador: um programa de genética clínica organizado e normatizado pelo Ministério da Saúde. Talvez a questão fundamental para a organização desta rede de serviços seja relacionada à gestão como um todo e estruturação dos financiamentos, respeitando as particularidades do atendimento na especialidade e a importância do fluxo de exames. Ao contrário do senso comum vigente, a genética não envolve necessariamente alto custo e alta tecnologia, sendo fundamental a atuação básica do especialista na área. Nesse sentido, a questão da organização da rede nada mais seria do que canalizar adequadamente os investimentos, e estruturar os serviços já existentes de uma maneira integrada e eficiente, levando a uma saúde mais justa e mais democrática. 


\section{Resumo}

O impacto dos defeitos congênitos no Brasil vem aumentando, apontando para a necessidade de estratégias específicas na política de saúde. Apesar da íntima ligação da genética clínica com a atenção aos defeitos congênitos, menos de $30 \%$ da demanda vem sendo absorvida pelos serviços do país. São problemas na atenção aos defeitos congênitos: dificuldades de acesso aos serviços de genética com concentração destes no Sul/Sudeste e suporte laboratorial insuficiente. Para melhor abordagem aos defeitos congênitos, ações para o estabelecimento de política em genética clínica deveriam ser deflagradas, preferencialmente sob coordenação de grupo técnico vinculado ao Ministério da Saúde, tendo como objetivo organizar rede clínico-laboratorial na especialidade. Ações visando à otimização de recursos e ao aumento da cobertura deverão ser consideradas. Para suporte laboratorial são prementes arranjos visando o fluxo de exames e criação de mecanismos de financiamento. Ações complementares de prevenção e registro epidemiológico dos defeitos congênitos, educação médica e do usuário são recomendadas. Com tais propostas contempladas, será possível a estruturação de uma rede regionalizada, hierarquizada e funcional, além de mais justa e mais democrática, voltada à atenção aos defeitos congênitos no Brasil.

Defeitos Congênitos; Genética Médica; Serviços em Genética; Planejamento em Saúde; Política de Saúde

\section{Colaboradores}

D. D. G. Horovitz contribuiu na pesquisa, elaboração e redação do artigo. M. H. C. A. Cardoso colaborou na elaboração e redação do artigo. J. C. Llerena Jr. e R. A. Mattos colaboraram na orientação e revisão da pesquisa.

\section{Referências}

1. Organização Pan-Americana da Saúde. Prevenção e controle de enfermidades genéticas e os defeitos congênitos: relatório de um grupo de consulta. Washington DC: Organização Pan-Americana da Saúde; 1984. (Publicação Científica, n. 460).

2. Powell-Griner E, Woolbright A. Trends in infant deaths from congenital anomalies: results from England and Wales, Scotland, Sweden and the United States. Int J Epidemiol 1990; 19:391-8.

3. Human Genetics Programme, World Health Organization. Community genetic services in Latin America and regional networks on medical genetics. Report of a WHO consultation. Geneva: World Health Organization; 2004.

4. Departamento de Informação e Informática do SUS. Sistema de Informações sobre Mortalidade - SIM. http://tabnet.datasus.gov.br/cgi/sim/obtmap.htm (acessado em 25/Out/2004).

5. Horovitz DDG. Atenção aos defeitos congênitos no Brasil: propostas para estruturação e integração da abordagem no sistema de saúde [Tese de Doutorado]. Rio de Janeiro: Instituto de Medicina Social, Universidade do Estado do Rio de Janeiro; 2003.

6. Horovitz DDG, Llerena Jr. JC, Mattos RA. Atenção aos defeitos congênitos no Brasil: panorama atual. Cad Saúde Pública 2005; 21:1055-64.

7. Brunoni D. Estado atual do desenvolvimento dos serviços de genética médica no Brasil. Rev Bras Genet 1997; 20 Suppl:11-23.

8. Acosta AX, Ferraz VEF. Exercício profissional em genética médica: genética clínica no Brasil. In: Anais do XII Congresso Brasileiro de Genética Clínica. Teresópolis: Sociedade Brasileira de Genética Clínica; 2000. p. 10.

9. Porciúncula CGG. Cadastro dos professores de genética dos cursos de medicina. In: Anais do XIII Congresso Brasileiro de Genética Clínica. Campinas: Sociedade Brasileira de Genética Clínica, 2001. p. 9.

10. Departamento de Informação e Informática do SUS. Sistema de Informações de Saúde. Sistema de Informações Sobre Nascimentos - SINASC. http://tabnet.datasus.gov.br/tabnet/tabnet. htm\#EstatVitais (acessado em 25/Out/2004).

11. Departamento de Informação e Informática do SUS. Sistema de Informações Ambulatoriais - SIA/ SUS. http://tabnet.datasus.gov.br/cgi/sia/pamap. htm (acessado em 31/Out/2003).

12. Departamento de Descentralização da Gestão da Assistência, Secretaria de Assistência à Saúde, Ministério da Saúde. Regionalização da assistência à saúde: aprofundando a descentralização com eqüidade no acesso. Norma Operacional da Assistência à Saúde - NOAS-SUS 01/02 (Portaria MS/GM n. 373, de 27 de fevereiro de 2002, e regulamentação complementar). $2^{\text {a }}$ Ed. Brasília: Ministério da Saúde; 2002. (Série A. Normas e Manuais Técnicos).

13. Peres E, Lauande R, Silva L, Souza I. Perfil dos pacientes atendidos no ambulatório de genética do hospital universitário Bettina Ferro de Souza da UFPA. In: Anais do XV Congresso Brasileiro de Genética Clínica. Porto Alegre: Sociedade Brasileira de Genética Clínica; 2003. p. 108. 
14. Faria APM, Ferraz VEF, Acosta AX, Brunoni D. Clinical genetics in the developing countries: the Brazilian situation. Community Genet 2004; 7:95-105.

15. Brunoni D. Aconselhamento genético. Ciênc Saúde Coletiva 2002; 7:101-7.

16. McKusick V. The growth and development of human genetics as a clinical discipline. Am J Hum Genet 1975; 27:261-73.

17. Hayflick SJ, Eiff MP. Role of primary care providers in the delivery of genetics services. Community Genet 1998; 1:18-22.

18. Boulyjenkov V. The role of the World Health Organization in promoting medical genetics in Latin America. Community Genet 2004; 7:70-3.

19. Qureshi N, Modell B, Modell M. Raising the profile of genetics in primary care. Nat Rev Genet 2004; 5:783-90.
20. Ministério da Saúde. Sistema de informações sobre nascidos vivos: manual de instruções para o preenchimento da declaração de nascido vivo. 3a Ed. Brasília: Ministério da Saúde; 1999.

21. Penchaszadeh VB, Christianson AL, Giugliani R, Boulyjenkov V, Katz M. Services for the prevention and management of genetic disorders and birth defects in developing countries. Community Genet 1999; 2:196-201.

22. Human Genetics Programme, Management of Noncommunicable Diseases, World Health Organization. Primary health care approaches for prevention and control of congenital and genetic disorders. Geneva: World Health Organization; 2000.

Recebido em 09/Jun/2005

Versão final reapresentada em 29/Mar/2006

Aprovado em 25/Abr/2006 